\title{
DOCUMENTO DIAGNÓSTICO INICIAL DE 11 E.S.E. EN LA APROPIACIÓN DE LA TELESALUD EN EL MODELO INTEGRAL DE ATENCIÓN EN SALUD DE TREINTA Y CUATRO (34) EMPRESAS SOCIALES DEL ESTADO
}

\author{
INITIAL DIAGNOSTIC DOCUMENT OF 11 E.S.E. IN THE \\ APPROPRIATION OF TELEHEALTH IN THE INTEGRAL MODEL OF \\ HEALTH CARE OF THIRTY-FOUR (34) SOCIAL STATE COMPANIES
}

\author{
Autor 1 - Magda Gioanna Cruz Suarez \\ Ms en Salud Pública y Desarrollo Social \\ Universidad Nacional Abierta y a Distancia \\ ORCID: https://orcid.org/0000-0001-6212-2968 \\ email.magda.cruz@unad.edu.co \\ Autor 2 - Paola Alvis Duffó \\ Esp. Seguridad y Salud en el Trabajo \\ Universidad Nacional Abierta y a Distancia \\ ORCID: https://orcid.org/0000-0001-6183-0137 \\ email.paola.alvis@unad.edu.co \\ Autor 3 - Myriam Leonor Torres Pérez \\ Phd. en Salud Pública \\ Universidad Nacional Abierta y a Distancia \\ ORCID: https://orcid.org/0000-0003-2923-5754 \\ email. Myriam.torres@unad.edu.co
}

\section{RESUMEN.}

El documento diagnóstico responde a la necesidad del fortalecimiento del "Proyecto Plan Fronteras para la Prosperidad" como estrategia del Gobierno Nacional a través de la Cancillería en aras de responder de manera oportuna a lo descrito en el Plan Nacional de Desarrollo 2010 2014 Prosperidad para todos, en el cual se define como un propósito nacional las áreas fronterizas, la promoción de políticas diferenciadas y la focalización de recursos, favoreciendo sectores estratégicos para el desarrollo de estas regiones. Por otro lado, contribuye a la gestión de la salud, mediante el desarrollo de las capacidades básicas para actuar en la planificación e interacción de las acciones relacionadas con la producción social de la salud dentro y fuera del sector. Así mismo la UNAD bajo el liderazgo de la Escuela de Ciencias de la Salud, y en 
corresponsabilidad con otros sectores y actores propone acciones para impactar positivamente los determinantes sociales y económicos de la salud, propiciando condiciones de vida, desarrollo social y económico sostenible en toda la población del país, incluidas las zonas fronterizas de manera transversal bajo los lineamientos definidos en el Plan Decenal de Salud Pública 2012-2021, en concordancia con la Política de Atención Integral en Salud y el Modelo Integral de Atención en Salud, entre otras políticas públicas vigentes.

\section{Palabras Clave.}

Telesalud; Apropiación; Habilitación; Biomédico.

\section{ABSTRACT.}

The diagnostic document responds to the need to strengthen the "Plan Borders for Prosperity Project" as a strategy of the National Government through the Foreign Ministry in order to respond in a timely manner to what is described in the National Development Plan 2010 - 2014 Prosperity for all, in which border areas, the promotion of differentiated policies and the targeting of resources, favoring strategic sectors for the development of these regions, are defined as a national purpose. On the other hand, it contributes to health management, through the development of basic capacities to act in the planning and interaction of actions related to the social production of health within and outside the sector. Likewise, UNAD under the leadership of the School of Health Sciences, and in co-responsibility with other sectors and actors, proposes actions to positively impact the social and economic determinants of health, promoting living conditions, social and economic development that is sustainable throughout the country's population, including cross-border areas under the guidelines defined in the Ten-Year Public Health Plan 2012-2021, in accordance with the Comprehensive Health Care Policy and the Comprehensive Health Care Model, among other public policies current.

\section{Keywords.}

Telehealth; Appropriation; Qualification; Biomedical. 


\section{INTRODUCCIÓN.}

La Universidad Nacional Abierta y a Distancia (UNAD) bajo el liderazgo de la Escuela de Ciencias de la Salud responde a la necesidad del fortalecimiento del "Proyecto Plan Fronteras para la Prosperidad" como estrategia del Gobierno Nacional a través de la Cancillería y en corresponsabilidad con otros sectores y actores propone acciones para impactar positivamente los determinantes sociales y económicos de la salud (1).

Por lo anterior, se desarrolla un proyecto que busca apropiar la telesalud y mejorar las oportunidades de acceso a servicios de salud, propiciando condiciones de vida, desarrollo social y económico sostenible en toda la población del país, incluidas las zonas fronterizas de manera transversal bajo los lineamientos definidos en el Plan Decenal de Salud Pública 20122021, en concordancia con la Política de Atención Integral en Salud y el Modelo Integral de Atención en Salud, entre otras políticas públicas vigentes $(2,3,4)$.

La Escuela de Ciencias de la Salud - ECISALUD y la Vicerrectoría de Medios y Mediaciones pedagógicas - VIMEP en representación de la UNAD, presentan el documento de diagnóstico inicial del estado de la Telemedicina en el 35\% correspondiente a las 11 de las 34 Empresas Sociales del Estado seleccionadas, el cual incluye información asociada a estado de habilitación en modalidad de atención telemedicina, conectividad, tecnología Biomédica y Recurso Humano disponible para la prestación de los servicios.

\section{METODOLOGÍA.}

La recolección de información se ejecutó a través de los datos suministrados por las E.S.E. y el análisis fue elaborado por la escuela de Ciencias de la Salud, pionera en la implementación de la Telemedicina en Colombia. Se realizaron actividades de recolección de información por personal de las E.S.E. y fue diligenciado un documento formato (5) con la información respectiva, recolectada y entregada por la misma E.S.E. en los siguientes aspectos:

1. Verificación Estado de Conectividad en las E.S.E.: Para evaluar el estado de conectividad se empleó como metodología la realización de pruebas test de velocidad de canal de internet en cada hospital desarrollado por personal de las E.S.E., las cuales permitieron identificar 
las características de cada enlace teniendo en cuenta los siguientes factores:

- Saturación.

- Simetría o asimetría del canal.

- $\quad$ Capacidad real del ancho de banda por canal medida en Mbps.

2. Verificación Estado de Habilitación de la modalidad de Telemedicina en las E.S.E.: Para validar el estado de habilitación de las E.S.E. se empleó como metodología la búsqueda a través del NIT y verificación de servicios habilitados en cada uno de los prestadores en la página web "Registro Especial de Prestadores de Servicios de Salud- REPS".

La verificación de información permitió identificar las siguientes características en cada E.S.E.:

- Tipo de Prestador Habilitado: Centro de referencia o Institución Remisora.

- $\quad$ Servicios Habilitados bajo la modalidad de Telemedicina.

3. Verificación Estado de Tecnología Biomédica en las E.S.E.: La verificación del estado de la tecnología biomédica y de cómputo para la prestación de telemedicina en las E.S.E. se realizó a través de la información suministrada por cada institución y el diligenciamiento del formato en el cual se evaluaron las siguientes condiciones de cada dispositivo:

- $\quad$ Lugar de ubicación del equipo

- Vigencia del Mantenimiento preventivo

- $\quad$ Estado físico y funcional de tecnología

4. Verificación Recurso Humano disponible para la prestación de los servicios de telemedicina en las E.S.E.: Los datos relacionados al recurso humano disponible para la prestación de los servicios de telemedicina se obtuvieron a través de la visita a cada ESE, indagando de manera 
telefónica en el área de talento humano acerca de la cantidad de personal de salud que labora en cada institución.

\section{DISCUSIÓN Y RESULTADOS.}

En torno a las 4 líneas de verificación desarrolladas en el 35\% de las E.S.E., en la verificación estado de conectividad, define como patrón de verificación y comparación 5 Mbps, ancho de banda mínima requerida para carga de estudios en una plataforma de telemedicina por cada E.S.E. De igual forma, el procedimiento se realizó tomando muestra para el reconocimiento del canal de internet con el que cuenta la institución y validación de la estabilidad del canal, todo esto se monitorea de forma remota.

A continuación, en el cuadro 1 se describen resultados de las pruebas realizadas:

Cuadro 1. Estado de la conectividad por E.S.E.

\begin{tabular}{|c|c|c|c|c|c|}
\hline \multirow{2}{*}{ E.S.E HOSPITAL } & \multicolumn{2}{|c|}{ INICIO } & \multicolumn{2}{|c|}{ FIN } & \multirow{2}{*}{$\begin{array}{l}\text { ESTADO DE } \\
\text { CONECTIVIDAD }\end{array}$} \\
\hline & Subida & Bajada & Subida & Bajada & \\
\hline E.S.E Hospital Local de Aguachica & 4,92 & 4,92 & 4,92 & 4,92 & Cumple \\
\hline $\begin{array}{l}\text { E.S.E Hospital Regional Noroccidente } \\
\text { Abrego }\end{array}$ & 2,21 & 1,89 & 1,83 & 2,84 & No cumple \\
\hline $\begin{array}{l}\text { E.S.E. Hospital Universitario Erasmo } \\
\text { Meoz }\end{array}$ & 2,9 & 2,8 & 2,9 & 2,9 & No cumple \\
\hline Hospital Cristian Moreno Pallares & 8,67 & 33,2 & 14 & 30 & Cumple \\
\hline $\begin{array}{l}\text { Hospital Inmaculada Concepción de } \\
\text { Chimichagua }\end{array}$ & 7,1 & 3.9 & 3,82 & 8,86 & Cumple \\
\hline Hospital Regional José Padilla Villafañe & 7,49 & 18,02 & 7,5 & 18 & Cumple \\
\hline Hospital San José de Becerril & 16,8 & 6,5 & 17,1 & 6,4 & Cumple \\
\hline E.S.E Cuspad Carlosama & 5,9 & 8,1 & 5,6 & 8,5 & Cumple \\
\hline E.S.E Hospital Cumbal & 14 & 66,2 & 14,5 & 67 & Cumple \\
\hline E.S.E Hospital Ricaurte & 1 & 0,5 & 1 & 0,5 & No cumple \\
\hline E.S.E Municipal de Ipiales & 0 & 0 & 0 & 0 & No cumple \\
\hline
\end{tabular}

Fuente: Elaboración propia

El análisis de datos encontrados permite clasificar el estado de entendiendo el cumplimiento como un estado adecuado para la prestación de telemedicina y transmisión optima de estudios por modalidad.

De acuerdo con lo anterior, se observa que el $64 \%$ de las 11 E.S.E. visitadas cumple con un estado de conectividad adecuado para prestar telemedicina y el $36 \%$ requieren acciones de mejora respecto al tema.

Respecto a los dos momentos de evaluación del canal: inicio y fin, se relacionan con el horario de atención de las E.S.E., por lo que según los datos encontrados se puede deducir que el canal de internet es mayor al finalizar el día, lo anterior asociado a la disminución del consumo de 
conectividad por los equipos tecnológicos de la institución y finalización de la jornada laboral de los trabajadores.

Para los casos no cumplimiento, se recomienda a la E.S.E. ampliar sus canales de conectividad con el fin de asegurar la transmisión de estudios por telemedicina y prestación de servicios.

En la Verificación Estado de Habilitación de la modalidad de Telemedicina en las E.S.E. Los datos encontrados indican entre los servicios habilitados bajo la modalidad de telemedicina en las 11 instituciones, los siguientes:

- Diagnóstico cardiovascular, el cual está asociado a apoyos diagnósticos y complementos terapéuticos como electrocardiograma, monitoreo ambulatorio de presión arterial, holter de ritmo y ecocardiograma, entre otros.

- $\quad$ Consulta Externa especialidades médicas, asociado a especialidades como Cardiología, Pediatría, Medicina Interna, Otorrinolaringología, entre otras.

- $\quad$ Radiología e imágenes diagnósticas de baja complejidad.

Los hallazgos permiten clasificar el estado de habilitación de las E.S.E. en tres categorías: cumple, parcial y no cumple, entendiendo el cumplimiento como la habilitación de dos o más servicios en la institución bajo la modalidad de telemedicina.

El cumplimiento parcial se refiere a la habilitación de al menos un servicio para prestación por telemedicina y el no cumplimiento indica que la E.S.E. no cuenta con ningún servicio inscrito en esta modalidad.

Cuadro 2. Estado de la Verificación

\begin{tabular}{|c|c|}
\hline ESTADO DE HABILITACIÓN & CANTIDAD E.S.E. \\
\hline CUMPLE & 5 \\
\hline PARCIAL & 3 \\
\hline NO CUMPLE & 3 \\
\hline
\end{tabular}

Fuente: Elaboración propia

De acuerdo con lo anterior, se observa que el $46 \%$ de las 11 E.S.E. cumple con los requisitos establecidos por la normatividad resolución 2003 de 2014 del Ministerio de Salud y Protección Social de Colombia respecto a las condiciones de inscripción y habilitación de servicios de salud en la modalidad de telemedicina.

El $27 \%$ de las 11 E.S.E., únicamente cuenta con un servicio habilitado por lo que se requiere ampliar información respecto a los servicios actualmente contratados para orientar el reporte de novedades de otros servicios en caso de requerirlo. 
El $27 \%$ de las 11 E.S.E. restante no cuenta con ningún servicio habilitado en telemedicina, lo cual requiere acciones de mejora respecto al proceso en el caso de prestar servicios a distancia.

Los resultados igualmente dan a conocer que el $100 \%$ de las 11 E.S.E. que cumplen con habilitación en la modalidad de telemedicina, ejecutaron el proceso de forma correcta ya que se encuentran inscritos como instituciones remisoras, es decir, instituciones prestadoras de salud que no brindan el servicio de forma presencial con el personal especializado de acuerdo con la situación clínica del individuo, sino que se apoyan para dar alcance a esta atención en un centro de referencia.

Según los resultados de la verificación de la información recolectada, de la tecnología relacionados con el lugar de ubicación de la tecnología, la visita permite reconocer que el $60 \%$ de los equipos se encuentran almacenados, lo que implica la exposición a partículas de polvo y condiciones ambientales que afectan el buen funcionamiento de la tecnología.

Se identifica que el $100 \%$ de los equipos requieren mantenimiento físico y lógico de la tecnología de cómputo y preventivo de los equipos biomédicos; de igual forma, se evidencia que en las instituciones no existe un responsable ni personal experto para el desarrollo de esta actividad.

Con relación a la funcionalidad de la tecnología, fue validado remotamente el estado de 95 equipos entre cómputo y biomédicos, formato Diagnóstico, lo anterior indica que no se logró validar la totalidad de equipos suministrados por el convenio implementado por el Plan Fronteras para la Prosperidad, esto debido a la falta de disponibilidad del personal de las E.S.E. para presentarlos y conocimiento de ubicación de estos.

Cuadro 3. Funcionalidad de la Tecnología

\begin{tabular}{|c|c|c|}
\hline TIPO DE EQUIPOS & FUNCIONALES & NO FUNCIONALES \\
\hline CÓMPUTO & 44 & 4 \\
\hline BIOMÉDICOS & 51 & 6 \\
\hline TOTAL & 95 & 9 \\
\hline
\end{tabular}

Fuente: Elaboración propia

Del total de equipos soportados en el formato de recolección de información, el $10 \%$ se encuentra no funcional, el restante aprobó las pruebas para prestar servicios de telemedicina.

Finalmente, la verificación del recurso humano disponible para la prestación de los servicios de telemedicina permite deducir que la zona de influencia de la E.S.E., es decir, su categoría regional, municipal o local 
está directamente relacionada con la cantidad de personal que labora en la institución.

Teniendo en cuenta lo anterior, del total de trabajadores de los hospitales, el $70 \%$ es personal asistencial y de estos, el $53 \%$ son médicos o enfermeros, lo que los define como recurso humano disponible para la prestación del servicio de telemedicina.

\section{CONCLUSIONES.}

Se realiza recolección de información asociada a estado de habilitación, conectividad, tecnología Biomédica y recurso Humano disponible para la prestación de servicios de telemedicina en el 35\% correspondiente a las 11 de las 34 Empresas Sociales del Estado que han sido beneficiadas en el marco del convenio implementado por el Plan Fronteras para la Prosperidad (1) que lidera el Ministerio de Relaciones Exteriores generando un documento de diagnóstico inicial del estado de condiciones que favorecen la telesalud en las instituciones, el cual será complementado en un diagnóstico consolidado de la totalidad de E.S.E. en una fase posterior.

Los datos obtenidos serán la línea base para el diseño de modelos de apropiación y fortalecimiento de la Telemedicina tanto en el componente de atención integral en salud como comercial en los diferentes prestadores.

\section{BIBLIOGRAFÍA.}

1. Ministerio de Relaciones Exteriores - Cancillería. Plan Fronteras para la prosperidad. 2013. Consultado febrero 2019. informeejecutivo-2013-plan-fronteras-la-prosperidad.pdf

(cancilleria.gov.co)

2. Ministerio de salud y protección Social Colombia. Plan Decenal de Salud Pública PDSP 2012-2021. 2012. Consultado febrero 2019. http://www.saludcapital.gov.co/DPYS/Documents/Plan\%20Decena \%20de\%20Salud\%20P\%C3\%BAblica.pdf

3. Ministerio de Salud y Protección Social. Política de Atención Integral en Salud. "Un sistema de salud al servicio de la gente". 2016. Consultado febrero 
https://www.minsalud.gov.co/sites/rid/Lists/BibliotecaDigital/RIDE LDE/modelo-pais-2016.pdf

4. Ministerio de Salud y Protección Social. Política de Atención Integral en Salud. Modelo Integral de Atención en Salud - MIAS. 2016. Consultado febrero 2019. https://www.minsalud.gov.co/sites/rid/Lists/BibliotecaDigital/RIDE /DE/01-pais-mias-capacitacion-sectorial.pdf

5. International Telemedical Systems Colombia S.A. Universidad Nacional Abierta y a Distancia. Formato Diagnóstico Estado Telemedicina Hospitales Proyecto "Apropiación Telesalud". 2018. 